\title{
Loss of JUNB/AP-1 promotes invasive prostate cancer
}

\author{
MK Thomsen ${ }^{1,3}$, L Bakiri ${ }^{1}$, SC Hasenfuss ${ }^{1,4}, \mathrm{H} \mathrm{Wu}^{1}$, M Morente $^{2}$ and EF Wagner ${ }^{\star, 1}$
}

Prostate cancer is a frequent cause of male death in the Western world. Relatively few genetic alterations have been identified, likely owing to disease heterogeneity. Here, we show that the transcription factor JUNB/AP-1 limits prostate cancer progression. JUNB expression is increased in low-grade prostate cancer compared with normal human prostate, but downregulated in highgrade samples and further decreased in all metastatic samples. To model the hypothesis that this downregulation is functionally significant, we genetically inactivated Junb in the prostate epithelium of mice. When combined with Pten (phosphatase and tensin homologue) loss, double-mutant mice were prone to invasive cancer development. Importantly, invasive tumours also developed when Junb and Pten were inactivated in a small cell population of the adult anterior prostate by topical Cre recombinase delivery. The resulting tumours displayed strong histological similarity with human prostate cancer. Loss of JunB expression led to increased proliferation and decreased senescence, likely owing to decreased $\mathrm{p} 16^{\mathrm{Ink} 4 \mathrm{a}}$ and $\mathrm{p} 21^{\mathrm{CIP} 1}$ in epithelial cells. Furthermore, the tumour stroma was altered with increased osteopontin and S100 calcium-binding protein A8/9 expression, which correlated with poor prognoses in patients. These data demonstrate that JUNB/AP-1 cooperates with PTEN signalling as barriers to invasive prostate cancer, whose concomitant genetic or epigenetic suppression induce malignant progression.

Cell Death and Differentiation (2015) 22, 574-582; doi:10.1038/cdd.2014.213; published online 19 December 2014

The incidence of prostate cancer is increasing in the Western world and patients with invasive disease have poor prognosis. ${ }^{1}$ Serum prostate-specific antigen (PSA) is used as a biomarker for cancer detection, but has shown limited predictive value for progression. ${ }^{2}$ Thus, there is an urgent need for improvements in both diagnosis and therapy. Presumably due to disease heterogeneity, very few genes have been identified that control progression of benign neoplasia to invasive prostate cancer. The use of mouse models has revealed that key genes and pathways like phosphatase and tensin homologue/protein kinase B (PTEN/ AKT), TRP53 and transforming growth factor beta/Smad are crucial for prostate cancer. ${ }^{3-6}$

The Activating Protein 1 (AP-1) transcription factor and its upstream kinases have been implicated in prostate cancer initiation/progression. ${ }^{7-11}$ c-Jun or c-Fos overexpression increased proliferation and invasiveness of prostate cancer cell lines and c-Fos, c-Jun and phosphorylated-c-Jun are increased in prostate cancer samples. ${ }^{8,10}$ In contrast, although the c-Jun $\mathrm{N}$-terminal kinase (JNK) signalling pathway is a functional target of PTEN, ${ }^{11}$ JNK ablation in mouse prostate epithelium leads to invasive prostate cancer, when combined with Pten loss. ${ }^{9}$ These studies indicate that although AP-1 members are implicated in prostate cancer, their functions might be stage- and context-specific.

JunB is a close homologue of c-Jun with tumoursuppressive function in the myeloid lineage,$^{12}$ which was proposed to be functionally relevant in human prostate cancer progression. ${ }^{13}$ Here, we show that JunB is downregulated in high-grade human prostate cancer. Moreover, using genetically modified mouse models, we demonstrate that although the loss of Junb in the prostate epithelium does not affect prostate homeostasis, combined loss of Junb and Pten even in a limited number of adult cells, leads to early-onset and invasive prostate cancer. Finally, we describe how JunB prevents the progression of invasive Pten-deficient prostate neoplasia by affecting both the tumour cells and the stroma.

\section{Results}

Decreased JUNB levels in prostate cancer progression. Prostate sections from prostatectomy samples were stained for JUNB expression by immunohistochemistry (IHC). As seen in Figure 1a, areas with normal prostate epithelia displayed low or undetectable JUNB, whereas areas with low-grade prostate cancer showed strong staining. Remarkably, and consistent with a previous report, ${ }^{13}$ JUNB was almost undetectable in the areas where the tumour had progressed to high grade (Figure 1a).

Next, JUNB mRNA expression was compared at different stages of prostate cancer using publicly available data sets. ${ }^{14-16}$ Strikingly, metastatic prostate cancer samples displayed significantly decreased JUNB mRNA expression in three independent data sets, when compared with primary

${ }^{1}$ Genes, Development and Disease Group, F-BBVA Cancer Cell Biology Programme, National Cancer Research Centre (CNIO), Madrid, Spain and ${ }^{2}$ Biobank, National Cancer Research Centre (CNIO), Madrid, Spain

${ }^{*}$ Corresponding author: E Wagner, Development and Disease Group, F-BBVA Cancer Cell Biology Programme, National Cancer Research Centre (CNIO), Melchor Fernandez Almagro 3, Madrid, 28029 Spain. Tel: +34 9122469 12; Fax:+34 9122469 14; E-mail: ewagner@cnio.es

${ }^{3}$ Current address: Department of Biomedicine, Aarhus University, Denmark.

${ }^{4}$ Current address: Dana-Farber Cancer Institute and Department of Cell Biology, Harvard Medical School, Boston, MA, USA.

Abbreviations: AP-1, activator protein 1; Pten, phosphatase and tensin homologue; PSA, prostate-specific antigen; TAC, transient-amplifying cell; ERK, extracellular signal-regulated kinase; AKT, protein kinase B; JNK, C-Jun N-terminal kinase; IHC, immunohistochemistry; SPP1, osteopontin; S100A8/9, S100 calcium-binding protein $\mathrm{A} 8 / 9$

Received 22.9.14; revised 17.11.14; accepted 19.11.14; Edited by G Melino; published online 19.12.14 
a

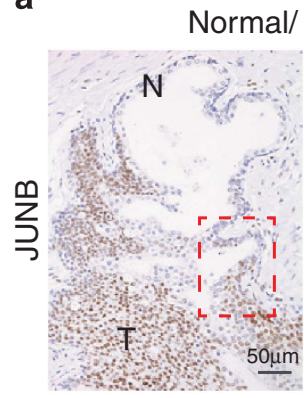

High grade

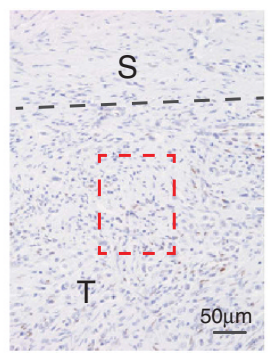

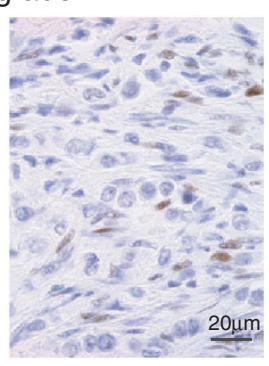

LaTulippe E, et al., b

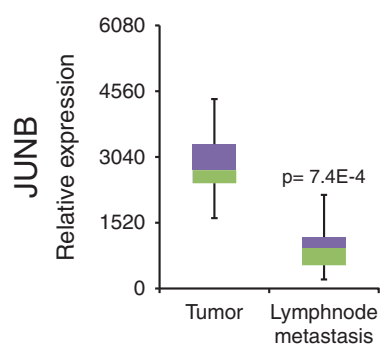

Chandran UR, et al.

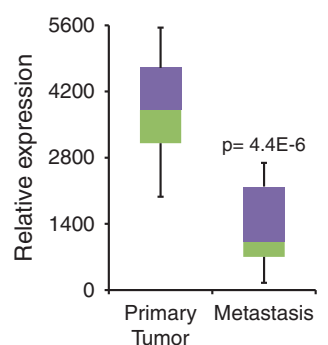

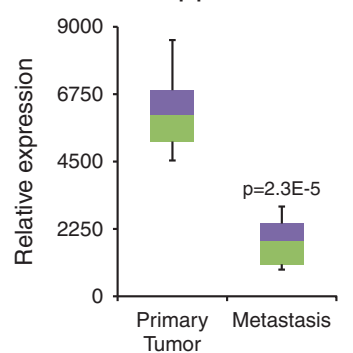

Figure 1 JUNB expression is decreased in high-grade and metastatic prostate cancer. (a) Tissue sections from prostatectomy samples were stained with an antibody to JUNB (brown). Representative area of low- and high-grade prostate cancer is shown from the same prostatectomy sample. Red box indicates the area of the high magnification. Arrowheads mark basal cells and dotted black line marks the border between high-grade cancer and the stoma. N (normal epithelia), T (tumour) and S (stoma); $n=8$. (b) JUNB expression from tumour and metastasis samples are shown from the data generated by ${ }^{16} 23$ tumour and 9 metastasis samples, ${ }^{14} 64$ tumour samples and 24 metastasis samples $a^{15} d^{15} 62$ tumour and 9 metastasis samples. Data were analyzed using Nextbio. $P$-values are indicated for each analysis

tumours (Figure 1b). Interestingly, decreased $c-J U N$ and c-FOS mRNA expression was also observed, whereas no consistent changes were noted for JUND, FRA1 and FRA2 across the three data sets (Supplementary Figure 1). Collectively, these data indicate that JUNB is downregulated, both at the mRNA and protein level, in aggressive prostate cancer.

JunB loss promotes invasive prostate cancer in mice lacking Pten. We next analyzed JunB expression in the $\operatorname{Pten}^{\triangle P}\left(\right.$ Pten $^{\text {flox/flox; }}$ PSA:Cre $\left.{ }^{T /+}\right)$ mouse model, where conditional inactivation of Pten is achieved by selective expression of re recombinase in the prostate epithelium. ${ }^{5,17}$ Consistent with our observations in human samples, IHC and mRNA expression revealed low to absent JunB in wild-type prostate and strong expression in prostatic intraepithelial neoplasia (PIN), which are low-grade neoplasia occurring in $\mathrm{Pten}^{\Delta \mathrm{P}}$ mutants (Figure 2a and Supplementary Figure 2a).

We next generated JunB ${ }^{\Delta P}$ mice, with specific inactivation of Junb in the prostate epithelium (JunB ${ }^{\text {flox/flox; }}$ PSA:Cre ${ }^{T /+}$ ). $J^{\prime} u n B^{\Delta P}$ mutant mice were viable and fertile and no histological alterations were observed in the prostate up to 9 months of age (Figure 2b), indicating that JunB is dispensable for prostate development and homeostasis.

To address the functional relevance of JunB in prostate cancer, JunB and Pten mutants were inter-crossed $\left(J u n B^{\text {flox/flox }} ;\right.$ Pten $^{\text {flox/flox; }}$ PSA:Cre $\left.{ }^{T /+}\right)$. The resulting JunB ${ }^{\Delta \mathrm{P}}$; $\operatorname{Pten}^{\Delta \mathrm{P}}$ double mutants developed prostate cancer as early as 8 weeks of age (Figure 2c). In addition, nearly $50 \%$ of double mutant mice showed invasive prostate cancer at 12 weeks of age, which was not observed in $\operatorname{Pten}^{\Delta \mathrm{P}}$ single mutants
(Figure 2d and Supplementary Figure 2b). Interestingly, although c-Jun expression was also increased in PIN from Pten $^{\Delta \mathrm{P}}$ mice (Supplementary Figure 2c), c-Jun ${ }^{\Delta \mathrm{P}}$; Pten $^{\Delta \mathrm{P}}$ (c-Jun ${ }^{\text {flox/flox; }}$ Pten flox/flox; PSA:Cre ${ }^{T /+}$ ) mice developed PIN with a similar latency as $\mathrm{Pten}^{\Delta \mathrm{P}}$ mice, and no invasive prostate cancer was observed (Supplementary Figure 2b). IHC confirmed the specific loss of c-Jun expression in prostate epithelium (Supplementary Figure 2c). These data indicate that JunB, but not c-Jun, prevents invasive prostate cancer in mice lacking Pten.

We, therefore, decided to focus our attention on investigating the function of JunB in prostate cancer. In-depth analyses revealed loss of basal cells and disrupted basal membranes in the invasive JunB ${ }^{\Delta P} ; \operatorname{Pten}^{\Delta P}$ double-mutant tumours, which is characteristic of human prostate cancer (Figure $2 \mathrm{e}$ and Supplementary Figures $2 \mathrm{~d}$ and e). IHC confirmed that JunB was similarly decreased in the prostate epithelium of invasive and noninvasive tumours, whereas stromal and immune cells remained JunB positive (Figure 2f). Molecular analyses revealed unaltered phosphorylation of AKT, extracellular signal-regulated kinase (ERK), p65 and comparable AP-1 and Smad4 gene expression between JunB ${ }^{\Delta P} ; \operatorname{Pten}^{\Delta P}$ and $P$ ten $^{\Delta P}$ tumours (Supplementary Figures $2 f$ and $h$ ). Interestingly, JNK phosphorylation appeared decreased in doublemutant tumours (Figure $2 \mathrm{~g}$ ), consistent with the reported function of JNK in invasive prostate cancer. ${ }^{9}$ These data indicate that JunB has an important role in the invasive progression of prostate neoplasia caused by Pten loss.

JunB loss leads to increased proliferation and decreased senescence. JunB has been shown to affect proliferation 
a

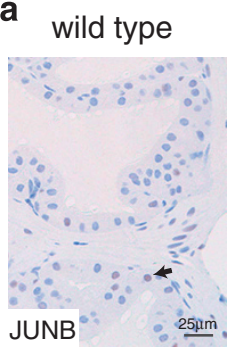

C

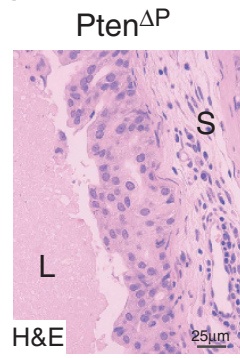

e

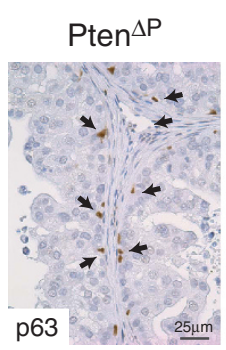

$\operatorname{Pten}^{\Delta \mathrm{P}}(\mathrm{PIN})$

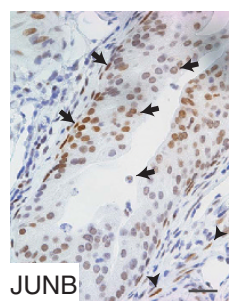

b
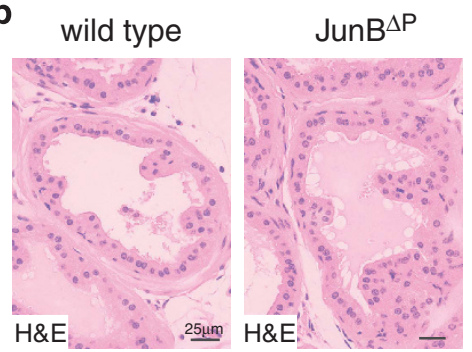

d
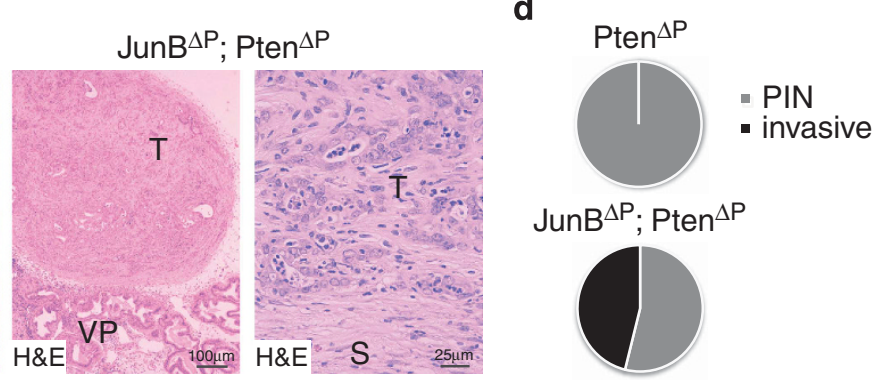

f

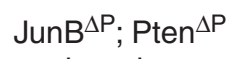
invasive

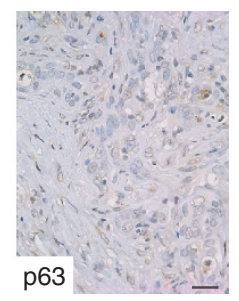

non invasive

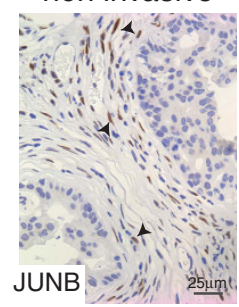

$\operatorname{JunB}^{\Delta \mathrm{P}} ; \mathrm{Pten}^{\Delta \mathrm{P}}$

invasive

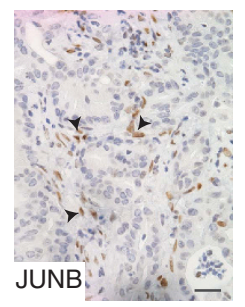

g

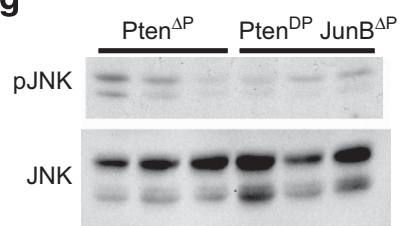

Figure 2 Loss of JUNB expression induces invasive prostate cancer. (a) IHC for JUNB in normal mouse dorsolateral prostate (wild type) and high-grade PIN (Pten ${ }^{\Delta P}$ : Pten $^{\text {floxfllox; }}$ PsaCre ${ }^{+T}$ ). Black arrows mark JUNB-positive cells in the epithelia. $n \geq 3$. A representative sample is shown. (b) Haematoxylin and eosin (H\&E) staining of dorsolateral prostate from wild-type, JunB ${ }^{\Delta \mathrm{P}}\left(\mathrm{JunB}^{\text {floxfllox; }}\right.$ PsaCre ${ }^{+/ T}$ ) mice; $n \geq 3$. A representative sample is shown. (c) H\&E staining of dorsolateral prostate from Pten ${ }^{\Delta \mathrm{P}}$ (Pten $^{\text {floxflox; }}$ PsaCre $^{+/ T}$ ) and JunB ${ }^{\Delta P} ;$ Pten $^{\Delta P}$ (Junb ${ }^{\text {floxfllox; }}$ Pten ${ }^{\text {floxfflox; }}$ PsaCre ${ }^{+/ T}$ ) mice. L (lumen), T (tumour), S (stroma) and VP (ventral prostate). $n \geq 3$. A representative sample is shown. (d) Incidence of invasive prostate cancer in 12-week-old mice. $n=\operatorname{Pten}^{\Delta P}: 0$ out of 12; JunB ${ }^{\Delta P} ; P^{2}{ }^{\Delta P}: 6$ out of 13. (e) Staining for basal cells with an antibody to p63 (brown). A representative noninvasive sample and an invasive sample are shown; $n=5$. (f) $I H C$ for JUNB in noninvasive and invasive prostate cancer from JunB ${ }^{\Delta P} ; P^{2}$ ten ${ }^{\Delta P}$ (Junb $^{\text {floxflox; }}$ Pten floxflox; PsaCre ${ }^{+/ T}$ ) mice. Black arrowheads mark JUNB-positive cells in the stroma. $n \geq 3$. A representative sample is shown. (g) Western blot for phospho-JNK in prostate samples from Pten ${ }^{\Delta P}$ and JunB ${ }^{\Delta P} ; P \operatorname{ten}^{\Delta P}$ mice. Total JNK is used as loading control; $n=3$

and senescence, ${ }^{18,19}$ two cellular processes relevant to prostate cancer. JunB $\mathrm{B}^{\Delta \mathrm{P}}$; Pten ${ }^{\Delta \mathrm{P}}$ double-mutant tumours had significantly more Ki67-positive proliferating cells in the noninvasive areas, when compared with $\mathrm{Pten}^{\Delta \mathrm{P}}$ tumours (Figure 3a,Supplementary Figure $3 a$ ) and this increase was maintained in the invasive areas (Figure $3 b$ ).

Transient-amplifying cells (TACs) area subset of basal cells from which prostate cancer is thought to originate and in human samples, JUNB was suggested to maintain TAC senescence. ${ }^{13}$ Although the number of TACs was not different between $P \operatorname{ten}^{\Delta P}$ and noninvasive JunB ${ }^{\Delta P} ;$ ten $^{\Delta P}$ tumours (Supplementary Figures $3 \mathrm{~b}$ and $\mathrm{c}$ ), the double-mutant tumours displayed weaker senescence-associated betagalactosidase activity (Figure $3 \mathrm{c}$ ). Furthermore, IHC revealed decreased $\mathrm{p} 16^{\text {Ink4a }}$ immune reactivity in JunB ${ }^{\Delta \mathrm{P}} ; \mathrm{Pten}^{\Delta \mathrm{P}}$ tumours, particularly in invasive areas (Figure $3 d$ ). Decreased p16 ${ }^{\text {Ink4a }}$, but also $\mathrm{p} 21^{\mathrm{CIP} 1}$, was confirmed by western blot analyses (Figure 3e).

Decreased p21 ${ }^{\mathrm{CIP} 1}$ expression between primary and metastatic prostate cancer was also observed in publicly available human patient data sets (Supplementary Figure 3f). Furthermore, p21 ${ }^{\mathrm{CIP} 1}$ and JUNB expressions positively correlated, suggesting an interaction between these two genes and prostate cancer progression (Figure 3f). Collectively, these 
a

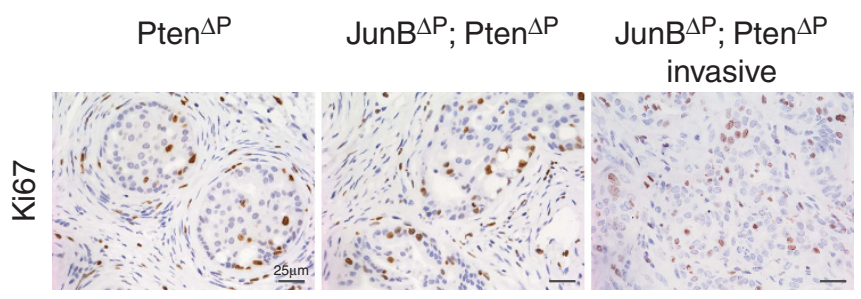

C
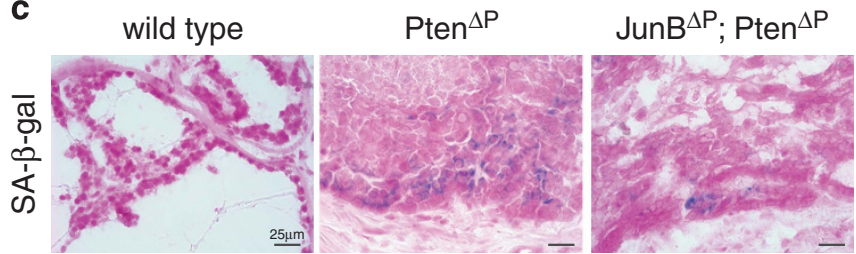

d

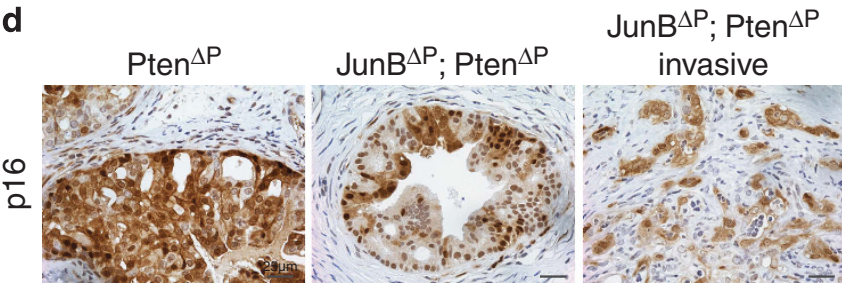

b

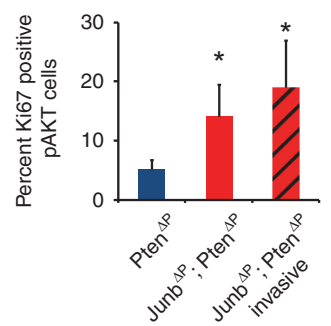

e

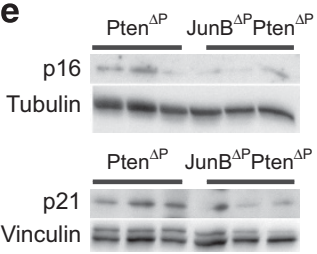

f

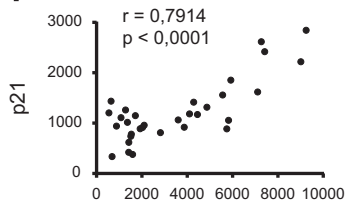

JUNB

Figure 3 JUNB represses proliferation and promotes senescence in prostate epithelial cells. (a) IHC for Ki67 in high-grade PIN and invasive dorsolateral prostate cancer from $\mathrm{Pten}^{\Delta \mathrm{P}}$ and JunB ${ }^{\Delta \mathrm{P}}$; Pten ${ }^{\Delta \mathrm{P}}$ mice as indicated. $n \geq 3$. A representative sample is shown. (b) Quantification of Ki67/ phospho-AKT double-positive cells from sections of dorsolateral prostate cancer from Pten ${ }^{\Delta P}$ and JunB $B^{\Delta P} ; P^{2} n^{\Delta P}$ mice. Three to four fields were counted from each sample and a total of $1800-3300$ cells were counted per group; $n=3-4$. ${ }^{*} P<0.05$. (c) Senescence-associated beta-galactosidase staining on frozen sections from the dorsolateral prostate; $n=3$. A representative sample is shown. (d) IHC for p16 in high-grade PIN and invasive prostate cancer from Pten ${ }^{\Delta \mathrm{P}}$ and JunB ${ }^{\Delta \mathrm{P}} ; \mathrm{Pten}^{\Delta \mathrm{P}}$ mice as indicated. $n \geq 3$. A representative sample is shown. (e) Western blots for p16 and p21 using prostate samples from Pten ${ }^{\Delta \mathrm{P}}$ and JunB ${ }^{\Delta \mathrm{P}}$; $\operatorname{Pten}^{\Delta \mathrm{P}}$ mice. Tubulin and vinculin were used as loading control; $n=3$. (f) Correlation plot for JUNB and $p 21 \mathrm{mRNA}$ expression from LaTulippe $\mathrm{E}$, et al. (23 tumour and 9 metastasis samples) bioset

data indicate that, both in mouse models and in patients, loss of JunB likely affects the proliferation and senescence of the neoplastic prostatic epithelium by decreasing $\mathrm{p} 16^{\text {Ink4a }}$ and $\mathrm{p} 21^{\mathrm{CIP} 1}$ expressions.

JunB loss in prostate epithelium affects the surrounding stroma. Enhanced expression of the calcium-binding proteins $\mathrm{S} 100$ calcium-binding protein $\mathrm{A} 8$ and A9 (S100A8 and S100A9) was observed in human prostate cancer, ${ }^{20}$ and Jun/AP-1 controls the S100a8/S100a9 expression in mouse epidermis. ${ }^{21,22}$ Increased expression of S100A8 and S100A9 was detected by western blot and IHC in JunB ${ }^{\Delta P}$; Pten $^{\triangle P}$ tumours, while mRNA expression was unchanged (Figure 4a and c). The S100A8- and S100A9-positive cells appeared to be non-epithelial and IHC co-staining with F4/80 indicated that most positive cells were of the monocyte/ macrophage lineage (Figure 4b, Supplementary Figure 4a). Surprisingly, pStat3 or $I I-1 b$ and $C c / 2$ expressions were not different between JunB ${ }^{\Delta \mathrm{P}} ;$ Pten $^{\Delta \mathrm{P}}$ and $\mathrm{Pten}^{\Delta \mathrm{P}}$ tumours (Supplementary Figure 4b).

Osteopontin (SPP1) is a part of the prognostic signature for human prostate cancer ${ }^{4}$ and is modulated by AP-1 in several tissues, such as the lung and liver. ${ }^{23,24}$ Consistent with the increased invasiveness, Spp1 mRNA was increased in JunB $^{\Delta P} ;$ Pten $^{\Delta P}$ tumours (Figure 4d). Interestingly, IHC analyses indicated that SPP1 was increased in invasive and noninvasive double-mutant tumours mainly in the stroma
(Figure 4e). Furthermore, expression of S100A8, S100A9 and SPP1 was increased in human metastatic prostate cancer samples and inversely correlated with JUNB was increased (Figure 4f). These data indicate that the loss of JunB in Ptendeficient prostate epithelium leads to increased S100A8, S100A9 and SPP1 in the stroma through a non-cellautonomous mechanism.

Targeting JunB and Pten in a limited cell number leads to invasive prostate cancer. We took advantage of an orthotopic viral Cre delivery approach ${ }^{25}$ to assess the genetic interactions between Pten and JunB in a clonal expansion model. Adeno-Cre was injected in the anterior prostate of 6-week-old Pten ${ }^{\text {flox/flox }}$ or JunB ${ }^{\text {flox/flox }}$; Pten ${ }^{\text {flox/flox }}$ mice and the resulting somatic mutants (Pten ${ }^{\Delta A d}$ and JunB ${ }^{\Delta A d}$; Pten ${ }^{\Delta A d}$ ) were analyzed 30 weeks later for tumour formation. Macroscopic lesions could be identified in the injected lobe of a subset of mutants (Figure 5a). Microscopic analysis revealed that although $\sim 50 \%$ of injected mice had formed lesions, lesion incidence was not related to the genotype. However, morphometric analysis indicated that JunB ${ }^{\Delta A d}$; Pten ${ }^{\Delta A d}$ lesions were significantly larger than $\operatorname{Pten}^{\Delta A d}$ lesions (Figure 5b). Furthermore, histological analysis revealed that half of the JunB ${ }^{\Delta A d}$; Pten ${ }^{\Delta A d}$ tumours were invasive, whereas none of the Pten ${ }^{\triangle A d}$ PIN had progressed (Figure $5 c$ and d). Increased proliferation was also apparent in $\mathrm{JunB}^{\triangle \mathrm{Ad} \text {; }}$ Pten $^{\Delta \mathrm{Ad}}$ lesions by Ki67 IHC (Figure 5e). IHC confirmed that 
a

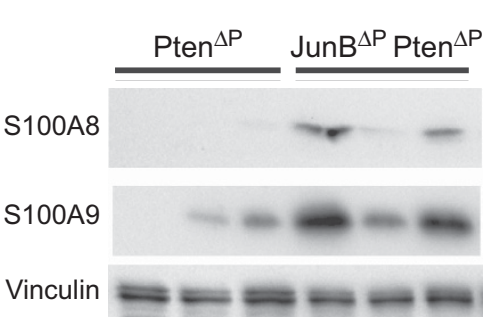

c

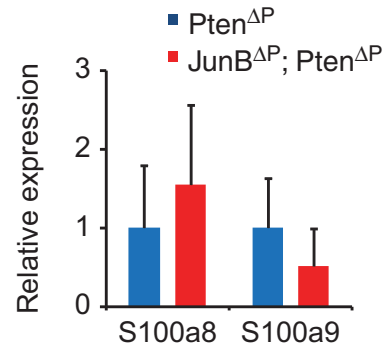

b
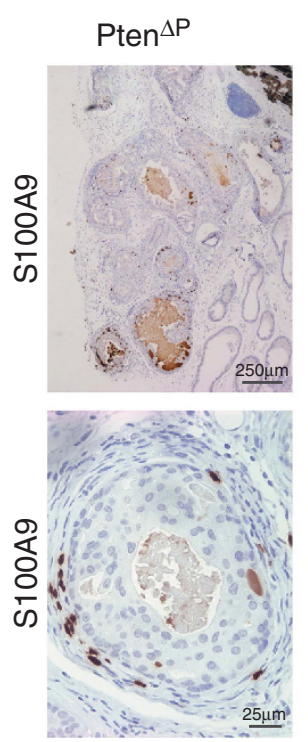

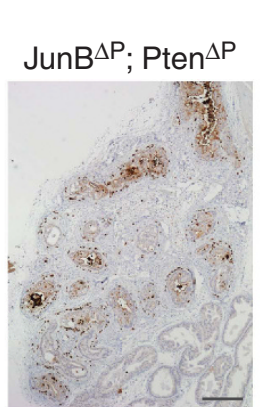

$\operatorname{JunB}^{\Delta \mathrm{P}} ;$ Pten $^{\Delta \mathrm{P}}$ invasive
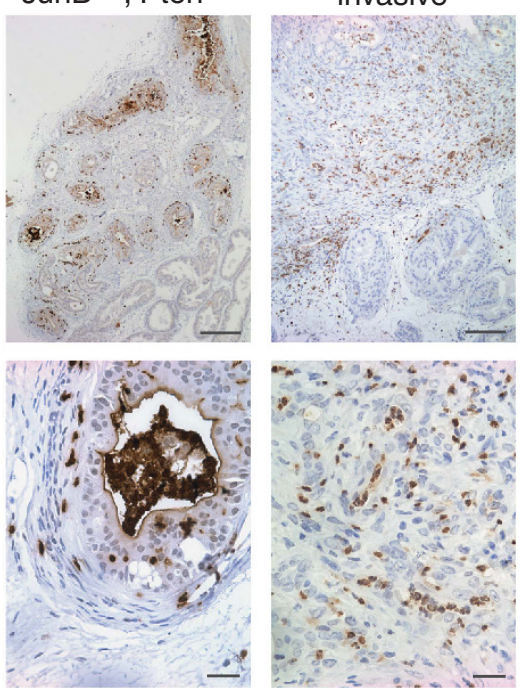

d

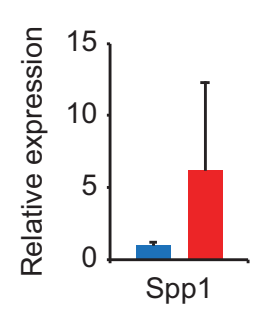

e

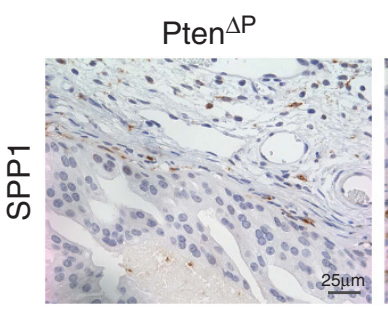

$\operatorname{JunB}^{\Delta P} ; \operatorname{Pten}^{\Delta P}$

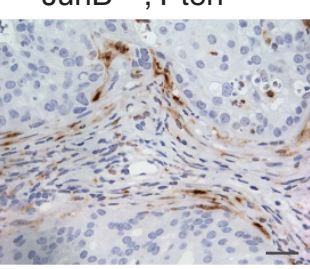

JunB $^{\Delta \mathrm{P}} ; \mathrm{Pten}^{\Delta \mathrm{P}}$

invasive

f
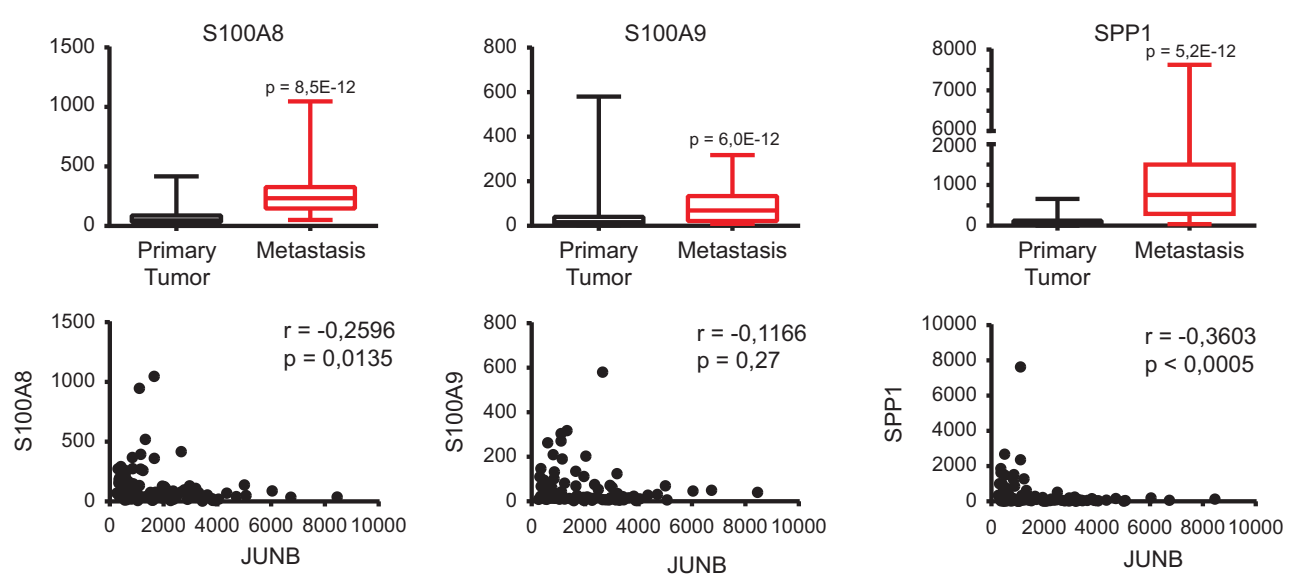

Figure 4 JUNB represses S100A8/9 and SPP1 in a non-cell-autonomous manner. (a) Western blots for S100A8 and S100A9 on prostate samples from Pten ${ }^{\Delta \mathrm{P}}$ and JunB $\mathrm{B}^{\Delta \mathrm{P}}$; $P \operatorname{Pten}^{\Delta \mathrm{P}}$ mice. Vinculin was used as a loading control; $n=3$. (b) IHC for S100A9 in high-grade PIN and invasive dorsolateral prostate cancer from Pten ${ }^{\Delta \mathrm{P}}$ and JunB ${ }^{\Delta \mathrm{P}} ; \mathrm{Pten}^{\Delta \mathrm{P}^{\prime}}$ mice as indicated. $n \geq 3$. A representative sample is shown. (c) Quantitative reverse transcription PCR (GRT-PCR) for S100A8 and S100A9 in prostate samples from Pten ${ }^{\Delta \mathrm{P}}$ and $J_{u n B^{\Delta P}}$; Pten ${ }^{\Delta P}$ mice. $n \geq 3$. (d) $q R T$-PCR for Spp1 in prostate samples from Pten ${ }^{\Delta P}$ and JunB ${ }^{\Delta P}$; $P$ ten ${ }^{\Delta P}$ mice. $n \geq 3$. (e) IHC for SPP1 in high-grade PIN and invasive prostate cancer from $P$ ten ${ }^{\Delta \mathrm{P}}$ and Jun $\mathrm{B}^{\Delta \mathrm{P}}$; Pten ${ }^{\Delta \mathrm{P}}$ mice as indicated. $n \geq 3$. A representative sample is shown. (f) Box-plot diagram for S100A8, S100A9 and SPP1 mRNA expressions and correlation with JUNB expression from Chandran et al. ${ }^{14}$ (64 tumour samples and 24 metastasis samples) bioset

the JunB expression was abolished in JunB ${ }^{\Delta A d} ; P_{t e n} \triangle A d$ tumours and all lesions stained positive for phospho-AKT regardless of the genotype (Figure 5f,Supplementary Figure 5a). The invasive JunB ${ }^{\Delta A d}$; Pten ${ }^{\triangle A d}$ lesions were lacking basal cells, consistent with the observations in the genetic inactivation model. In addition, areas with invasive basal cells and TACs were identified (Supplementary Figure 5b).
IHC showed reduced $\mathrm{p} 16^{\text {Ink4a }}$ in JunB ${ }^{\Delta A d}$; Pten ${ }^{\Delta A d}$ tumours when compared with Pten ${ }^{\triangle A d}$ lesions, whereas SPP1, S100A8 and S100A9 expressions were increased in the stroma (Supplementary Figure 6). Overall, these results show that the combined loss of JunB and Pten in a subset of adult prostate epithelial cells is sufficient to promote invasive cancer. 
a

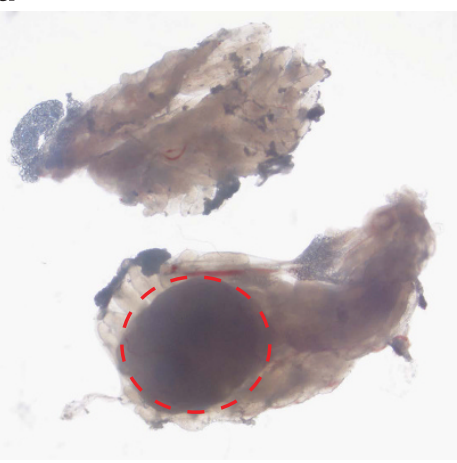

d

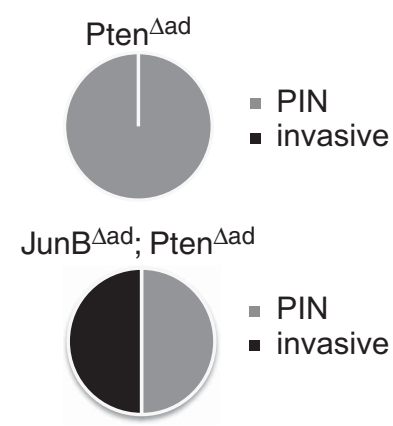

b

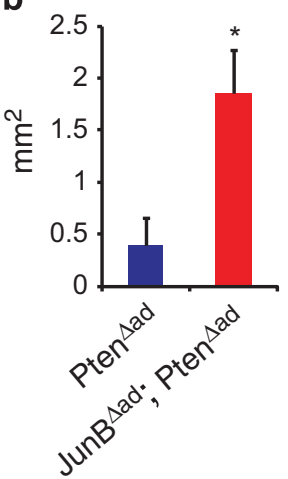

C

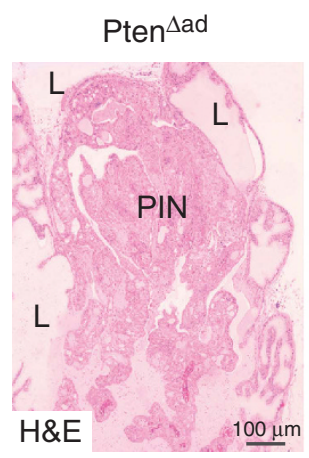

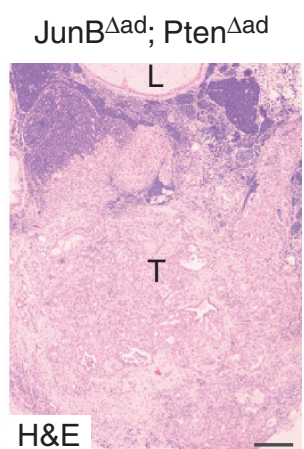

Junb $^{\Delta \text { ad; }}$ Pten ${ }^{\Delta \text { ad }}$ invasive

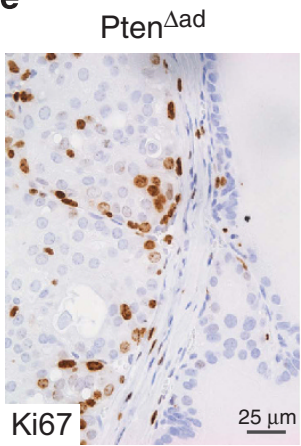

Junb $^{\Delta \text { ad }} ;$ Pten $^{\Delta a d}$

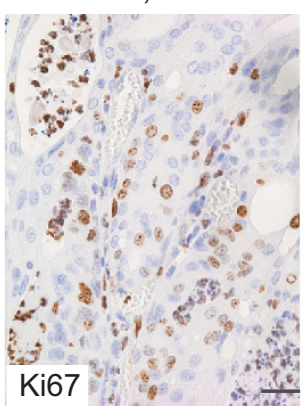

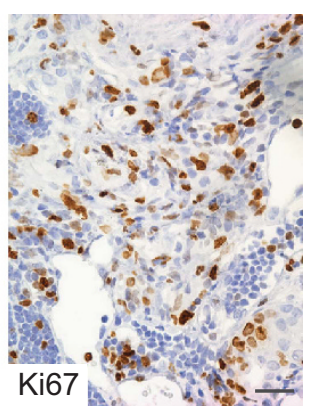

Junb $^{\Delta \text { ad }} ;$ Pten $^{\Delta \text { ad }}$ invasive

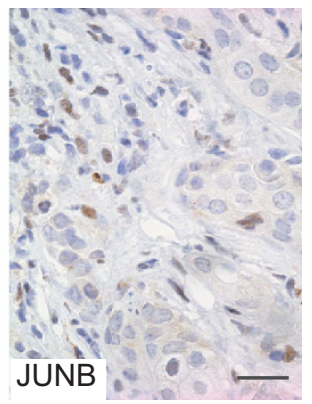

Figure 5 Loss of JUNB and PTEN in single cells leads to invasive prostate cancer. Throughout the whole figure, all mice carrying floxed alleles for Pten or Junb and Pten were injected with $10^{8} \mathrm{pfu}$ Adeno-Cre into an anterior lobe at 6 weeks of age. Mice were killed for analysis at 36 weeks of age. (a) Macroscopic image of the anterior prostate 30 weeks after injection of Adeno-Cre. The red doted circle marks the area of the lesion; $n=12$. A sample with invasive prostate cancer is shown. (b) Quantification of the lesions by measurement of the horizontal and vertical length time $0.8 ; n=4$ and $P<0.05$. (c) Haematoxylin and eosin staining of the anterior prostate from Pten ${ }^{\Delta a d}$ and JunB ${ }^{\Delta a d}$; Pten ${ }^{\Delta a d}$ samples as indicated. L (lumen), PIN and T (tumour); $n=6$. A representative sample is shown. (d) Graph shows the incidents of invasive prostate cancer 30 weeks post deletion; $n=$ Pten $^{\Delta \text { ad. }} 0$ out of 6 ; JunB ${ }^{\Delta \text { ad; }}$; Pten ${ }^{\Delta a d}$ : 3 out of 6. (e) IHC for Ki67 in high-grade PIN and invasive prostate cancer from Pten ${ }^{\Delta a d}$ and JunB ${ }^{\Delta a d}$; Pten ${ }^{\Delta a d}$ mice as indicated; $n \geq 3$. A representative sample is shown. (f) IHC for JUNB in normal, high-grade PIN and invasive prostate cancer from wild-type, Pten ${ }^{\Delta a d}$ and JunB ${ }^{\Delta a d}$; Pten ${ }^{\Delta a d}$ mice as $^{2}$ indicated; $n \geq 3$. A representative sample is shown

\section{Discussion}

Dysregulation of the PTEN/AKT pathway is the most frequent event in prostate cancer ${ }^{26}$ and it is, therefore, important to identify the cofactors modulating prostate cancer progression in the context of altered PTEN/AKT signalling. Mouse models with specific loss of Pten in combination with other genes have unravelled prostate cancer modulators, such as TRP53, SMAD4 and JNK. 4,6,9,3,27-29 We show here that JunB limits tumour progression of Pten-deficient prostate neoplasia as the loss of Junb, even in few cells, is sufficient to promote invasiveness.
In human samples, JUNB protein and mRNA expression was decreased in advanced and metastatic disease. Interestingly, we additionally observed that JUNB was upregulated in human but also in mouse prostate low-grade hyperplasia, when compared with normal prostate epithelium. This suggests that JunB could have a dual function, promoting the development of benign lesions and limiting subsequent invasion. However, inactivating JunB in the prostate by two independent approaches revealed that JunB is dispensable for PIN development, at least in the context of Pten loss, as the incidence of prostate tumours was not altered upon additional 
inactivation of Junb. In addition, although the combined inactivation of Pten and JunB elicited invasive prostate cancer, JunB was found dispensable for prostate development and no tumours developed in single Junb-deficient mice.

Intriguingly, our bioinformatic analyses of human prostate cancer data sets revealed decreased c-Jun mRNA in metastatic samples. Increased c-Jun protein and phosphorylated-c-Jun is documented in prostate cancer samples and is associated with worse patient outcome. 8,10 This would indicate that, unlike JunB, the regulation of c-Jun expression in human prostate cancer is largely post transcriptional and rather supports an oncogenic function for c-Jun. C-Jun was also increased in Pten ${ }^{\Delta P}$ PIN samples. In addition, no significant differences in tumorigenesis were observed, when c-Jun was inactivated in $\operatorname{Pten}^{\Delta \mathrm{P}}$ mice and no invasive prostate cancer was observed up to 6 months of age. Although we cannot exclude subtle and minor differences given the number of mice examined, c-Jun appears dispensable for the development of low-grade neoplasia in ten $^{\Delta \mathrm{P}}$ mutants.

Prostate cancer is proposed to arise from a single clone from which sub-clones can emerge. ${ }^{30-32}$ We took advantage of an orthotopic adenovirus-based, Cre delivery approach ${ }^{25}$ to model the human scenario. Targeting few epithelial cells in the prostate was sufficient to trigger invasive prostate cancer, when both Junb and Pten were inactivated. Thus, two independent ablation methods led to the same result and increased the invasiveness of Pten-deficient prostate epithelial cells. It should be noted that pathological examination of the lungs and lymph nodes did not reveal metastatic cells in any of the mutant mice, possibly owing to the relatively short observation period.

Loss of Junb resulted in increased proliferation of Pten-deficient prostate cells and this increase was independent of invasiveness. Decreased expression of the cell cycle inhibitors $\mathrm{p} 21^{\mathrm{CIP} 1}$ and $\mathrm{p} 16^{\text {lnk4a }}$ was consistently observed in JunB/Pten-deficient tumours, and $\mathrm{p} 21^{\mathrm{CIP} 1}$ and JUNB expressions correlated in human samples. Increased proliferation was accompanied by a decrease in cellular senescence, a process that opposes cancer progression upon Pten loss. ${ }^{33}$ Thus, Junb can be added to the limited list of genes, such as Trp53, ${ }^{6} \mathrm{Jnk}^{9}$ and Smad $4,{ }^{4}$ which allow bypassing senescence and promoting tumourigenesis in Pten-deficient PIN.

Although inactivation of the Ink4a/Arf locus, encoding both p16 $6^{\text {Ink4a }}$ and p19 ${ }^{\text {Arf }}$, leads to accelerated PIN development in Pten $^{+/-}$mice, ${ }^{34}$ combined deletion of Pten and p19 Arf in prostate epithelium does not accelerate prostate cancer. ${ }^{35}$ This indicates that $\mathrm{p} 16^{\mathrm{lnk} 4 \mathrm{a}}$ is the relevant gene in the Ink4a/Arf locus for Pten-associated prostate cancer. In vitro experiments using primary human TACs suggested that JUNB maintains TAC senescence by increasing $p 16^{\text {Ink } 4 a}$ mRNA, ${ }^{13}$ consistent with the modulation of proliferation, senescence and $p 16^{\text {Ink4a }}$ transcription by JunB in mouse fibroblasts. ${ }^{19,36}$ How the lack of JunB leads to decreased $\mathrm{p} 16^{\mathrm{Ink} 4 \mathrm{a}}$ and $\mathrm{p} 21^{\mathrm{CIP} 1}$ proteins in JunB/Pten-deficient lesions in vivo with no apparent effect on mRNA remains to be determined. Differences in JunBmediated transcriptional regulation between the distinct cell types, as already documented for $p 16^{\text {Ink4a }}$ and cyclind 1 in hematopoietic cells, ${ }^{37}$ could explain this discrepancy. It is also possible that changes in other transcriptional regulators and/ or signalling pathways counterbalance the direct effect of JunB on $p 16^{\text {Ink4a }}$ transcription in prostate epithelial cells, while promoting post-transcriptional effects. One pathway could involve JNK, as phospho-JNK was decreased in JunB/Ptendeficient lesions and JNK deficiency leads to comparable changes in the proliferation and senescence in Pten-deficient PIN. ${ }^{9}$

Interestingly, although loss of JunB expression did not alter the number of infiltrating immune cells, in particular F4/80positive cells (data not shown), the loss of JUNB led to increased SPP1 and S100A8/A9 expressions in the stroma, particularly in monocytes/macrophages. As SPP1 and S100A8/A9 are associated with aggressive prostate cancer with poor prognosis, ${ }^{4,20}$ the mechanistic basis of the cross-talk between the tumour and stroma in this model warrants further investigations. Likewise, although AKT signalling has been shown to modulate JunB/AP-1 expression and activity in vitro, ${ }^{38}$ studying the mechanism by which JunB/AP-1 is downregulated in human patient samples through genetic/ epigenetic or environmental cues should be at centre stage for future experiments.

In conclusion, our mouse genetic data establish JunB as a novel gate keeper for prostate cancer progression in the context of pten loss. As PTEN and its downstream pathway are commonly lost or dysfunctional in human prostate cancer, our findings are likely of relevance for the development of diagnostic and therapeutic strategies.

\section{Materials and Methods}

Human samples. Prostate samples originate from prostatectomy. Four samples from well-differentiated samples with a Gleason score of 5-6 and four samples from advanced adenocarcinoma with Gleason 8-9 and perineural invasion were used for the study.

Mice and treatments. Junb $b^{\text {floxflox }}, c-J u n^{\text {floxfllox }}$, Pten ${ }^{\text {floxflox }}$ and PSA:Cre mice have been previously described. ${ }^{5,17,39,40}$ Mice were maintained on a mixed C57Bl6x129sv background. Mice were killed at 8-12 weeks and age-matched littermates were used. Pten ${ }^{\text {floxflox }}$ or Junb ${ }^{\text {floxfllox; }}$; Pten ${ }^{\text {floxflox }}$ mice were injected with $10^{8}$ pfu Adeno-Cre (lowa University) virus in $25 \mu \mathrm{l}$ PBS at 6 weeks of age..$^{25}$ Analysis for lesions 30 days later showed none to three small focal areas of pAKTpositive cells (data not shown). All experiments were stopped 30 weeks after virus injection and the prostate, lymph nodes and lungs were harvested for analysis.

Immunohistochemistry. Tissues were fixed in 4\% PFA and embedded in paraffin. Haematoxylin and eosin staining was performed according to the standard procedures. Antigen retrieval was performed in a pressure cooker using citrate buffer at pH6. The following antibodies were used for IHC: JunB (CS3753), pAKT (CS4060), Ki67 (Dako, Barcelona, Spain; SP1 (rabbit) or TEC-3 (rat)), CK5 (PRB-160P, Covance, Madrid, Spain), CK8 (Troma1, Developmental Studies Hybridoma Bank (DSHB), University of lowa, lowa City, IA, USA), p16 (SC-1661), S100A9 (SC-8115), SPP1 (R\&D Systems, Madrid, Spain; AF808), smooth muscle actin (SMA, Sigma A2547, Madrid, Spain) and F4/80 (Biolegend, Madrid, Spain). The appropriate secondary antibodies coupled with FITC455, AlexaFluorF594 or HRP were used for fluorescence or coupled with a chromogenic staining. Counterstaining was performed using DAPI or haematoxylin.

Senescence. Tissues were washed through a sucrose gradient and frozen in OCT. Eight-micron sections were cut and dried at $37^{\circ} \mathrm{C}$ for $1 \mathrm{~h}$ before being washed with PBS for three times. Tissues were fixed for $10 \mathrm{~min}$ and incubated overnight with the staining solution (CS9860) at $37^{\circ} \mathrm{C}$, according to the manufacturer's instructions.

Protein isolation and western blots. Tissues were disrupted using a Precellys 24 device (Bertin, Lyon, France) in RIPA buffer containing a protease inhibitor cocktail (Sigma), $0.1 \mathrm{mM} \mathrm{Na} \mathrm{NO}_{4}, 40 \mathrm{mM}$ B-glycerophosphate, 
$40 \mathrm{mM} \mathrm{NaPPi}, 1 \mathrm{mM} \mathrm{NaF}$. Approximately $50 \mu \mathrm{g}$ of protein per sample were loaded for western blot analysis and loading was eventually adjusted using actin (Sigma, A-2066) and vinculin (Sigma, V-9131). The membranes were blocked with $2.5 \%$ BSA in TBS-T before being incubated with the primary antibody. The following antibodies were used: pJNK (CS9251), c-Jun (CS9165), JNK (CS9252), pAKT (CS4060), AKT (CS9272) pErk1/2 (CS9101), p-p65 (CS3031) p65 (SC-372), p16 (SC-1207), p21 (BD556430), pStat3 (CS3131), Stat3 (CS9132), S100A8 (R\&D Systems, AF3059) and S100A9 (R\&D Systems, AF2065). Appropriate HRP-linked secondary antibodies were purchased from Amersham and DAKO.

Quantitative reverse transcription PCR. RNA was isolated with Trizol (Sigma) and complementary DNA synthesized with Ready-To-Go-You-Prime-FirstStrand Beads (GE Healthcare, Madrid, Spain), using $1 \mu \mathrm{g}$ of DNAse-pretreated total RNA and random hexamers. Quantitative PCR was performed using GoTaq qPCR Master Mix (Promega, Madrid, Spain) and an Eppendorf fluorescence thermocycler. The comparative cycle threshold method was used for quantification. Expression levels were normalized using at least one housekeeping gene (actin, gapdh). Primer sequences are available upon request.

Data mining. Prostate bioset from Chandran et al.. ${ }^{14}$ Lapointe et al. ${ }^{15}$ and LaTulippe et al. ${ }^{16}$ were analysis in NextBio. Chandran et al. ${ }^{14}$ has 64 tumour samples and 24 metastasis samples. LaTulippe et al. ${ }^{16}$ has 23 tumour and 9 metastasis samples. Lapointe et al. ${ }^{15}$ (2004) has 62 tumour and 9 lymph node metastasis samples. Gene expressions from primary prostate cancer were compared with metastatic-derived prostate cancer. $P$-values were generated by NextBio analysis base on Student's t-test. Published array data analyses were used to generated box-plot and correlations analysis.

Statistical analyses. All experiments were performed at least three times and data in bar graphs represent mean \pm S.D. of the indicated sample numbers. Statistical analysis was performed using non-directional two-tailed Student's $t$-test. ${ }^{*} P<0.05$ was considered as significant.

Ethical study approval. Paraffin sections of prostate tissues originating from prostatectomy were provided by the CNIO tumour bank in accordance with the ethical guidelines of the Helsinki Declaration. Mouse handling and experimentation was done in accordance with local and institutional guidelines and regulations.

\section{Conflict of Interest}

The authors declare no conflict of interest.

Acknowledgements. We are very grateful to $G$. Luque and $G$. Medrano for technical help with mouse procedures. This work was supported by the Banco Bilbao Vizcaya Argentaria Foundation (F-BBVA), the Spanish Ministry of Economy (Grant BFU2012-40230), the Association for International Cancer Research (AICR/13-0216) and a European Research Council-advanced grant (ERC-FCK/2008/37) to EFW. MKT was supported by a Juan de la Cierva postdoctoral fellowship. SCH was the recipient of a Boehringer Ingelheim Fonds PhD fellowship and a European Molecular Biology Organization short-term fellowship (ASTF 198-2012).

1. Siegel R, Naishadham D, Jemal A. Cancer statistics. CA Cancer J Clin 2013; 63: 11-30.

2. Liu Y, Hegde P, Zhang F, Hampton G, Jia S. Prostate cancer - a biomarker perspective Front Endocrinol (Lausanne) 2012; 3: 72.

3. Parisotto M, Metzger D. Genetically engineered mouse models of prostate cancer. Mol Oncol 2013; 7: 190-205.

4. Ding Z, Wu CJ, Chu GC, Xiao Y, Ho D, Zhang J et al. SMAD4-dependent barrier constrains prostate cancer growth and metastatic progression. Nature 2011; 470: 269-273.

5. Wang S, Gao J, Lei Q, Rozengurt N, Pritchard C, Jiao J et al. Prostate-specific deletion of the murine Pten tumor suppressor gene leads to metastatic prostate cancer. Cancer Cell 2003; 4: 209-221.

6. Chen Z, Trotman LC, Shaffer D, Lin HK, Dotan ZA, Niki M et al. Crucial role of p53-dependent cellular senescence in suppression of Pten-deficient tumorigenesis. Nature 2005; 436 725-730.

7. Chen SY, Cai C, Fisher CJ, Zheng Z, Omwancha J, Hsieh CL et al. c-Jun enhancement of androgen receptor transactivation is associated with prostate cancer cell proliferation. Oncogene 2006; 25: 7212-7223

8. Edwards J, Krishna NS, Mukherjee R, Bartlett JM. The role of c-Jun and c-Fos expression in androgen-independent prostate cancer. J Pathol 2004; 204: 153-158.
9. Hubner A, Mulholland DJ, Standen CL, Karasarides M, Cavanagh-Kyros J, Barrett T et al. JNK and PTEN cooperatively control the development of invasive adenocarcinoma of the prostate. Proc Natl Acad Sci USA 2012; 109: 12046-12051.

10. Ouyang X, Jessen WJ, Al-Ahmadie H, Serio AM, Lin Y, Shih WJ et al. Activator protein-1 transcription factors are associated with progression and recurrence of prostate cancer. Cancer Res 2008; 68: 2132-2144.

11. Vivanco I, Palaskas N, Tran C, Finn SP, Getz G, Kennedy NJ et al. Identification of the JNK signaling pathway as a functional target of the tumor suppressor PTEN. Cancer Cell 2007; 11: $555-569$.

12. Passegue E, Jochum W, Schorpp-Kistner M, Mohle-Steinlein U, Wagner EF. Chronic myeloid leukemia with increased granulocyte progenitors in mice lacking junB expression in the myeloid lineage. Cell 2001; 104: 21-32.

13. Konishi N, Shimada K, Nakamura M, Ishida E, Ota I, Tanaka N et al. Function of JunB in transient amplifying cell senescence and progression of human prostate cancer. Clin Cancer Res 2008; 14: 4408-4416.

14. Chandran UR, Ma C, Dhir R, Bisceglia M, Lyons-Weiler M, Liang W et al. Gene expression profiles of prostate cancer reveal involvement of multiple molecular pathways in the metastatic process. BMC Cancer 2007; 7: 64 .

15. Lapointe J, Li C, Higgins JP, van de Rijn M, Bair E, Montgomery K et al. Gene expression profiling identifies clinically relevant subtypes of prostate cancer. Proc Natl Acad Sci USA 2004; 101: 811-816

16. LaTulippe E, Satagopan J, Smith A, Scher H, Scardino P, Reuter V et al. Comprehensive gene expression analysis of prostate cancer reveals distinct transcriptional programs associated with metastatic disease. Cancer Res 2002; 62 : 4499-4506.

17. Ma X, Ziel-van der Made AC, Autar B, van der Korput HA, Vermeij M, van Duijn P et al. Targeted biallelic inactivation of Pten in the mouse prostate leads to prostate cancer accompanied by increased epithelial cell proliferation but not by reduced apoptosis. Cancer Res 2005; 65: 5730-5739.

18. Bakiri L, Lallemand D, Bossy-Wetzel E, Yaniv M. Cell cycle-dependent variations in c-Jun and JunB phosphorylation: a role in the control of cyclin D1 expression. EMBO J 2000; 19: 2056-2068.

19. Passegue E, Wagner EF. JunB suppresses cell proliferation by transcriptional activation of p16(INK4a) expression. EMBO J 2000; 19: 2969-2979.

20. Hermani A, Hess J, De Servi B, Medunjanin S, Grobholz R, Trojan L et al. Calcium-binding proteins S100A8 and S100A9 as novel diagnostic markers in human prostate cancer. Clin Cancer Res 2005; 11: 5146-5152.

21. Zenz R, Eferl R, Kenner L, Florin L, Hummerich L, Mehic D et al. Psoriasis-like skin disease and arthritis caused by inducible epidermal deletion of Jun proteins. Nature 2005; 437 : 369-375.

22. Schonthaler HB, Guinea-Viniegra J, Wculek SK, Ruppen I, Ximenez-Embun P, Guio-Carrion A et al. S100A8-S100A9 protein complex mediates psoriasis by regulating the expression of complement factor C3. Immunity 2013; 39: 1171-1181.

23. Eferl R, Hasselblatt $\mathrm{P}$, Rath M, Popper H, Zenz R, Komnenovic V et al. Development of pulmonary fibrosis through a pathway involving the transcription factor Fra-2/AP-1. Proc Natl Acad Sci USA 2008; 105: 10525-10530.

24. Hasenfuss SC, Bakiri L, Thomsen MK, Hamacher R, Wagner EF. Activator Protein 1 transcription factor Fos-related antigen 1 (Fra-1) is dispensable for murine liver fibrosis, but modulates xenobiotic metabolism. Hepatology 2014; 59: 261-273.

25. Leow CC, Wang XD, Gao WQ. Novel method of generating prostate-specific Cre-LoxP gene switching via intraductal delivery of adenovirus. Prostate 2005; 65: 1-9.

26. Reid AH, Attard G, Ambroisine L, Fisher G, Kovacs G, Brewer D et al. Molecular characterisation of ERG, ETV1 and PTEN gene loci identifies patients at low and high risk of death from prostate cancer. Br J Cancer 2010; 102: 678-684

27. Francis JC, McCarthy A, Thomsen MK, Ashworth A, Swain A. Brca2 and Trp53 deficiency cooperate in the progression of mouse prostate tumourigenesis. PLOS Genet 2010; 6 : e1000995.

28. Francis JC, Thomsen MK, Taketo MM, Swain A.. beta-catenin is required for prostate development and cooperates with Pten loss to drive invasive carcinoma. PLoS Genet 2013. 9: e1003180

29. Thomsen MK, Ambroisine L, Wynn S, Cheah KS, Foster CS, Fisher G et al. SOX9 elevation in the prostate promotes proliferation and cooperates with PTEN loss to drive tumor formation. Cancer Res 2010; 70: 979-987.

30. Gerlinger M, Rowan AJ, Horswell S, Larkin J, Endesfelder D, Gronroos E et al. Intratumor heterogeneity and branched evolution revealed by multiregion sequencing. $N$ Engl $\mathrm{J} \mathrm{Med}$ 2012; 366: 883-892

31. Haffner MC, Mosbruger T, Esopi DM, Fedor H, Heaphy CM, Walker DA et al. Tracking the clonal origin of lethal prostate cancer. J Clin Invest 2013; 123: 4918-4922.

32. Liu W, Laitinen S, Khan S, Vihinen M, Kowalski J, Yu G et al. Copy number analysis indicates monoclonal origin of lethal metastatic prostate cancer. Nat Med 2009; 15: 559-565.

33. Nardella C, Clohessy JG, Alimonti A, Pandolfi PP. Pro-senescence therapy for cancer treatment. Nat Rev Cancer 2011; 11: 503-511.

34. You MJ, Castrillon DH, Bastian BC, O'Hagan RC, Bosenberg MW, Parsons R et al. Genetic analysis of Pten and Ink4a/Arf interactions in the suppression of tumorigenesis in mice. Proc Natl Acad Sci USA 2002; 99: 1455-1460. 
35. Chen Z, Carracedo A, Lin HK, Koutcher JA, Behrendt N, Egia A et al. Differential p53-independent outcomes of p19(Arf) loss in oncogenesis. Sci Signal 2009; 2: ra44.

36. Yogev $\mathrm{O}$, Anzi S, Inoue K, Shaulian E.. Induction of transcriptionally active Jun proteins regulates drug-induced senescence. J Biol Chem 2006; 281: 34475-34483.

37. Santaguida M, Schepers K, King B, Sabnis AJ, Forsberg EC, Attema JL et al. JunB protects against myeloid malignancies by limiting hematopoietic stem cell proliferation and differentiation without affecting self-renewal. Cancer Cell 2009; 15: 341-352.
38. Vartanian R, Masri J, Martin J, Cloninger C, Holmes B, Artinian N et al. AP-1 regulates cyclin D1 and c-MYC transcription in an AKT-dependent manner in response to mTOR inhibition: role of AIP4/Itch-mediated JUNB degradation. Mol Cancer Res 2011; 9: 115-130.

39. Kenner L, Hoebertz A, Beil FT, Keon N, Karreth F, Eferl R et al. Mice lacking JunB are osteopenic due to cell-autonomous osteoblast and osteoclast defects. J Cell Biol 2004; 164 613-623.

40. Behrens A, Sibilia M, David JP, Mohle-Steinlein U, Tronche F, Schutz G et al. Impaired postnatal hepatocyte proliferation and liver regeneration in mice lacking $\mathrm{c}$-jun in the liver. EMBOJ 2002; 21: 1782-1790.

Supplementary Information accompanies this paper on Cell Death and Differentiation website (http://www.nature.com/cdd) 